\title{
… \\ Preparation of NbAs Single Crystal by the Seed Growth Process
}

\author{
Yinchang Sun ${ }^{\dagger}$, Bojin Zhao ${ }^{\dagger}$, Zongju Huo, Hongjun Liu, Yongkuan Xu, Zhanggui Hu and Hailong Qiu *(D) \\ Tianjin Key Laboratory of Functional Crystal Materials, Institute of Functional Crystal, \\ Tianjin University of Technology, Tianjin 300384, China; acencore@163.com (Y.S.); zbojin@stud.tjut.edu.cn (B.Z.); \\ huozongju@stud.tjut.edu.cn (Z.H.); hjliu@email.tjut.edu.cn (H.L.); orientallion@126.com (Y.X.); \\ hu@tjut.edu.cn (Z.H.) \\ * Correspondence: qiu@tjut.edu.cn \\ t The authors contribute equally to this work.
}

check for updates

Citation: Sun, Y.; Zhao, B.; Huo, Z.; Liu, H.; Xu, Y.; Hu, Z.; Qiu, H. Preparation of $\mathrm{NbAs}$ Single Crystal by the Seed Growth Process. Crystals 2022, 12, 249. https://doi.org/ $10.3390 /$ cryst12020249

Academic Editors: Chunhui Yang, Ning Ye and Haohai Yu

Received: 19 January 2022

Accepted: 9 February 2022

Published: 11 February 2022

Publisher's Note: MDPI stays neutral with regard to jurisdictional claims in published maps and institutional affiliations.

Copyright: (c) 2022 by the authors. Licensee MDPI, Basel, Switzerland. This article is an open access article distributed under the terms and conditions of the Creative Commons Attribution (CC BY) license (https:// creativecommons.org/licenses/by/ $4.0 /)$.

\begin{abstract}
A Weyl semimetal is a novel crystal with low-energy electronic excitations that behave as Weyl fermions. It has received worldwide interest and was believed to have introduced the next era of condensed matter physics after graphene and three-dimensional topological insulators. However, it is not easy to obtain a single large-sized crystal because there are many nucleations in the preparation process. A bottom-seed CVT growth method is proposed in this paper, and we acquired the large-sized, high-quality $\mathrm{NbAs}$ single crystals up to $4 \times 3 \times 3 \mathrm{~mm}^{3}$ finally. X-ray diffraction and STEM confirmed that they are tetragonal $\mathrm{NbAs}$, which the key is to using the seed crystal in a vertical growth furnace. Notably, the photoelectric properties of the crystal are obtained under the existing conditions, which paves the way for follow-up work.
\end{abstract}

Keywords: Weyl semimetal; NbAs single crystal; seed growth; photoelectric

\section{Introduction}

Weyl semimetals (WSMs) have obvious chiral fermions and spin structures that can cause a novel carrier response under pulsed laser excitation, leading to new optical phenomena [1-5]. Because of these characteristics, WSMs have been favored by many scientists in recent years. Furthermore, according to the photodetection theory, semiconductors, which have bandgaps smaller than the photo energy of incident light [6], can have their electrons excited from valance bands to conduction bands to achieve a photoresponse. Therefore, WSM materials have excellent development potential in designing and applying new devices [7-9]. NbAs is a typical WSM.

$\mathrm{NbAs}$ is one of the first-class Weyl semimetals (TaAs, TaP, NbAs, $\mathrm{NbP}$ ), which crystallizes in a body-centered tetragonal unit cell with lattice constants $a=3.452 \AA, c=11.679 \AA$, and the space group is $14{ }_{1} m d\left(\mathrm{C}_{\frac{11}{4 v}}\right)[10,11]$ (as shown in Figure 1a). For NbAs, the melting point of $\mathrm{Nb}$ reaches $2468^{\circ} \mathrm{C}$, while the As (melting point: $817^{\circ} \mathrm{C}$ ) has the characteristics of direct sublimation without liquefaction when heated to $613^{\circ} \mathrm{C}$ under pressure. Therefore, a high-pressure environment is very favorable for the reaction conditions of As [12]. At the same time, regarding $\mathrm{NbAs}$, as a compound containing As, for safety reasons, the open growth system cannot meet the requirements of the growth environment. Besides $\mathrm{NbAs}$, other phases, such as $\mathrm{NbAs}_{2}, \mathrm{Nb}_{3} \mathrm{As}, \mathrm{Nb}_{4} \mathrm{As}_{3}$, exist in the $\mathrm{Nb}$-As binary system. Therefore, in reports, the chemical vapor transport (CVT) method is usually used to grow large-sized, high-quality $\mathrm{NbAs}$ single crystals [13].

Unfortunately, there are always many nucleations in this process during the growth of $\mathrm{NbAs}$. We believe that there are two reasons for this. On the one hand, the transmission capacity of the raw material atmosphere is limited. On the other hand, the quartz tube's axial and radial temperature gradients are large $[14,15]$. As a result, the environment of the raw material atmosphere during crystal growth is uneven and unstable, and the continuity of crystal growth cannot be maintained. Finally, small polycrystalline grains are obtained, 
which is very unfavorable to the growth of large-sized crystals. This paper provides a bottom-seed crystal growth process, which solves the above problems. Firstly, the vertical CVT process growth method is used; the raw materials are placed at the bottom of the quartz tube [16]. In this case, the raw material concentration at the bottom of the quartz tube will be much greater than that at the top, which is conducive to the growth of crystals at the bottom of the quartz tube when the transport distance of gas is significantly shortened. Secondly, the volume of the growth zone used for vertical growth will be much smaller than that of the horizontal CVT method, thus reducing the axial and radial temperature gradients in the growth zone and further ensuring the stability and continuity of crystal growth. In addition, the crystal with the exposed surface of (001) is added as the seed crystal, which provides a growth site for the raw material atmosphere and reduces the barrier of crystal growth. Thus, we believe that these methods are the key to obtaining large-sized and high-quality crystals $[17,18]$.

(a)

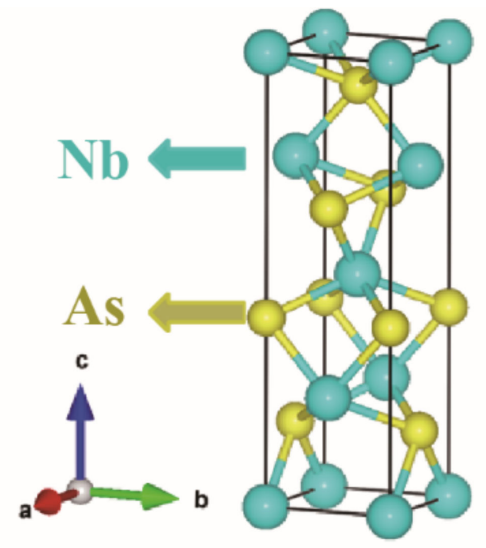

(c)

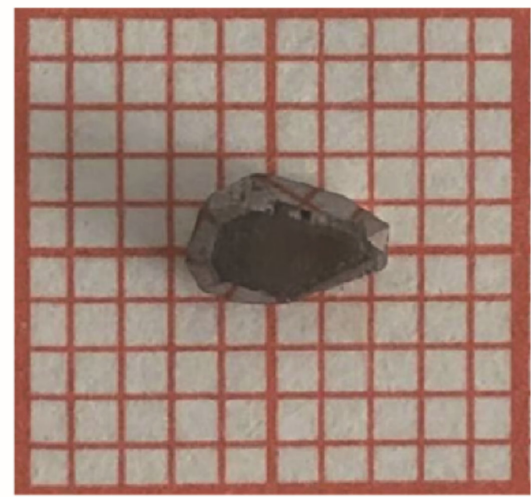

(b)

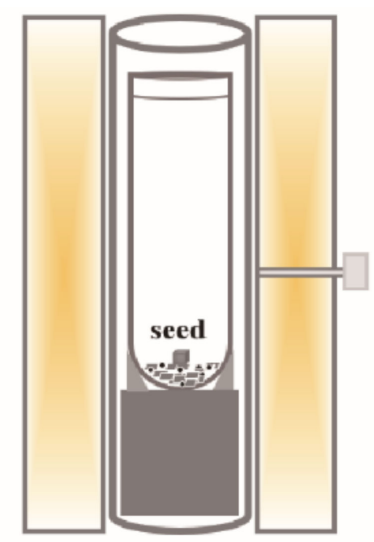

(d)

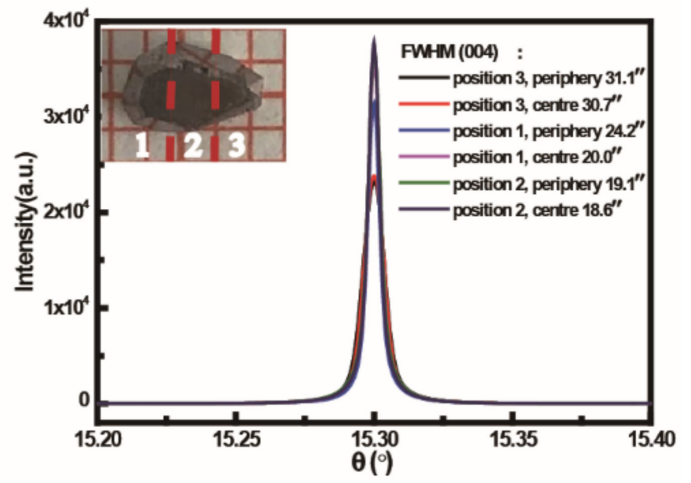

Figure 1. (a) Crystal structure of NbAs. (b) Schemes of the chemical vapor transport experiments for crystallization of $\mathrm{NbAs}$ in a two-step method. (c) Photograph of NbAs single crystal. (d) X-ray rocking curves of the three different regions (as shown in the illustration) for (004) face show good crystallinity with Full Width at Half Maxima (FWHM) less than 31.1".

\section{Materials and Methods}

\subsection{Synthesis of $\mathrm{NbAs}$ Crystal}

As is well known, exploring an appropriate growth process is the key to obtaining large crystals. We report a bottom-seed CVT growth method in this work, and Figure $1 \mathrm{~b}$ shows the experimental configurations. In a typical run, $\mathrm{Nb}$ foil (99.99\%), As (99.99\%), and $\mathrm{I}_{2}(99.99 \%)$ with the molar ratio of 1:1.05:0.05 were selected as raw materials, loaded in a $45 \mathrm{~mL}$ quartz tube, which was $14 \mathrm{~cm}$ in length and $2 \mathrm{~cm}$ in inner diameter. Meanwhile, we selected the grain of $\mathrm{NbAs}$ with a classy crystal orientation (here, the $\{001\}$ facets were selected as the growth section) as the seed; then, it was added into the quartz tube together with the raw materials. 
The quartz tube was initially filled with argon and sealed quickly to avoid the loss of iodine and arsenic when evacuated to a pressure below $4 \times 10^{-4} \mathrm{~Pa}$. $\mathrm{Nb}$ powder and $\mathrm{Nb}$ foil have a larger specific surface area and few defects, which is conducive to acquiring large crystals [11]. In addition, Saini et al [19] found that As must be excessive if we wish to obtain the best crystal growth effect, even for the growth of metal-rich crystals in the study of the Pd-As system. Therefore, here, we added a slight excess of As to achieve the growth of the $\mathrm{NbAs}$ single crystal. As for $\mathrm{I}_{2}$, it plays a catalytic and transport role according to the kinetic analysis of $\mathrm{I}_{2}$ in the growth of a TaAs crystal by Li [12] et al. Moreover, to avoid the explosion of the quartz tube caused by the vapor pressure generated when $\mathrm{I}_{2}$ and As are sublimated to a gaseous form, the amount of $I_{2}$ selected was $1 \mathrm{mg} / \mathrm{ml}$ here.

Afterward, the quartz tube was heated gradually from room temperature to $1000{ }^{\circ} \mathrm{C}$ over $72 \mathrm{~h}$, kept for 30 days in a high-temperature environment at $1050^{\circ} \mathrm{C}$, and finally naturally cooled down to room temperature (although the reaction time is longer, after comparing the growth results many times, a long-term heat preservation process was found to be essential). Through the above work, we obtained $\mathrm{NbAs}$ single crystals with good crystallinity, with a size of up to $4 \times 3 \times 3 \mathrm{~mm}^{3}$ in three dimensions (as Figure $1 \mathrm{c}$ shows). However, we have not seen a giant $\mathrm{Nb}$ As single-crystal reported to date. In addition, the X-ray rocking curves (Figure 1d) showed that the full widths at half maximum (FWHM) were less than 31.1" for the three different regions (as shown in the illustration) for the (004) face, suggesting the high quality of the grown crystals in terms of crystallinity.

\subsection{Device Fabrication}

$\mathrm{Nb}$ As devices are produced by dry transfer technology under argon filling conditions. The $\mathrm{NbAs}$ crystal size is close to $2 \mathrm{~mm} \times 1.5 \mathrm{~mm} \times 1.5 \mathrm{~mm}$ cubed (length $\times$ width $\times$ height). Since the sample size was relatively small, we did not choose the spin coating Ag adhesive method. We chose to attach the tape to the (001) plane of the NbAs crystal (the parameters of the sample crystal plane were determined by XRD) and left blank boundaries on both sides. Then, the sample surface was fully evaporated by evaporation. At the end of the evaporation, the tape attached to the surface was carefully torn apart so that the tape attached to the sample surface became the rectangular channel of the device. Both sides were conductive electrodes: $\mathrm{Cr} / \mathrm{Au}(10 \mathrm{~nm} / 70 \mathrm{~nm})$. The reason for choosing a $\mathrm{Cr} / \mathrm{Au}$ electrode is that the stability of the $\mathrm{Cr} / \mathrm{Au}$ electrode material deposited by evaporation is relatively good. In addition, the $\mathrm{Cr} / \mathrm{Au}$ evaporation deposition electrode can overcome the possible problems of poor contact and unstable conductivity of the $\mathrm{Ag}$ and $\mathrm{Cu}$ conductive adhesive spin coating. Finally, the prototype image of the $\mathrm{NbAs}$ photodetector is shown in Figure 2.

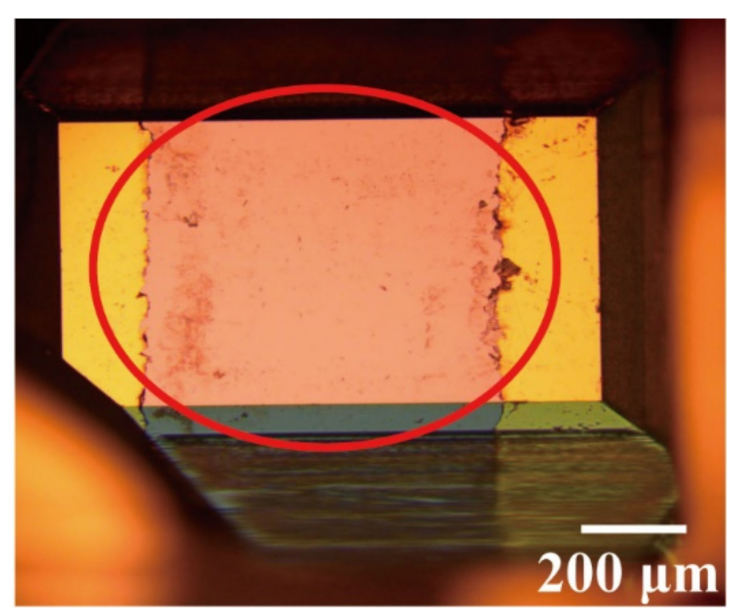

Figure 2. Device pictures of $\mathrm{NbAs}$ photodetectors. 
Semiconductor Parameter Analyzer (Keithley-4200-SCS, Bradford, UK) and standard probe station are earmarks of electronic and photoelectric measurement. The photoresponse of the device was measured using a laser with adjustable power and incident wavelength.

Powder X-ray Diffraction (PXRD). The PXRD measurements were collected using a SmartLab3KW X-ray diffractometer (Rigaku, Tokyo, Japan) at room temperature ( $\mathrm{Cu} \mathrm{K} \alpha$ radiation). All the data were collected in the $2 \theta$ range of $20-70^{\circ}$ with a step size of $0.01^{\circ}$ and a step time of $2 \mathrm{~s}$.

Raman Spectroscopy. The Raman spectra were collected from the single crystals of $\mathrm{Nb}$ As on a confocal microscope laser Raman spectrometer (WITec, Munich, Germany) equipped with a CCD detector using $532 \mathrm{~nm}$ radiation. The Raman data collection was accomplished in $20 \mathrm{~s}$.

X-Ray Photoelectron Spectroscopy (XPS). The XPS was performed on a Thermo Scientific (Waltham, MA, US) device, ESCALAB250Xi, with a monochromatic Al X-ray source. The power was $150 \mathrm{~W}$, and the spot size was 300 micrometers.

Scanning Transmission Electron Microscopy (STEM). The sample was prepared using the standard polymer transfer method. STEM images and HAASF-STEM were obtained using FEI, TECNAI G2Spirit TWIN (Thermo Scientific Waltham, MA, US).

Energy Dispersive X-ray Spectroscopy (EDX). Microprobe elemental analyses and the elemental distribution map were performed using an energy-dispersive $\mathrm{X}$-ray spectroscope (EDX) scanning transmission electron microscopy (Thermo Scientific Waltham, MA, US). The average atomic ratio in $\mathrm{NbAs}$ was $\mathrm{Nb}$ : $\mathrm{As}=49.72$ : 50.28, which was approximately equal to the theoretical one, 1:1.

\section{Results and Discussion}

\subsection{Structure Analysis}

Niobium arsenide, $\mathrm{NbAs}$, crystallizes in a body-centered tetragonal Bravai lattice, space group $I 4_{1} m d$, point group $\mathrm{C}_{4 \mathrm{v}}$. Our X-ray diffraction (XRD) obtained lattice constants of $a=3.45 \AA$ and $c=11.68 \AA$, consistent with earlier crystallographic studies. In Figure 3a, the upper black line in the diagram indicates the XRD of $\mathrm{NbAs}$ powder (obtained by grinding NbAs crystals), which is completely consistent with the data in PDF No. 17-0896 (blue line in the figure). In addition, we checked the main peak positions and marked the corresponding crystal planes. Among them, a few noncorresponding crystal planes belonged to polyarsenides, marked with the yellow graphic (such as $\mathrm{NbAs}_{2}$ ) [20-22], and their existence is temporarily unavoidable in the growth of this type of crystal. The result shows that the grown $\mathrm{NbAs}$ crystals have high purity and few miscellaneous items.

Note that, for the seed of NbAs, its exposed surface is mainly $\{001\}$ facets, and the one-dimensional length is approximately $1.5 \mathrm{~mm}$. These faces have a relatively larger lattice plane space and, therefore, a slower growth rate and thus are found more frequently than others in the as-grown crystals [12]. Nevertheless, when we use these seed crystals for further growth, they can provide a basis for the growth of other planes in the NbAs crystal so that other poorly developed planes can be displayed. 

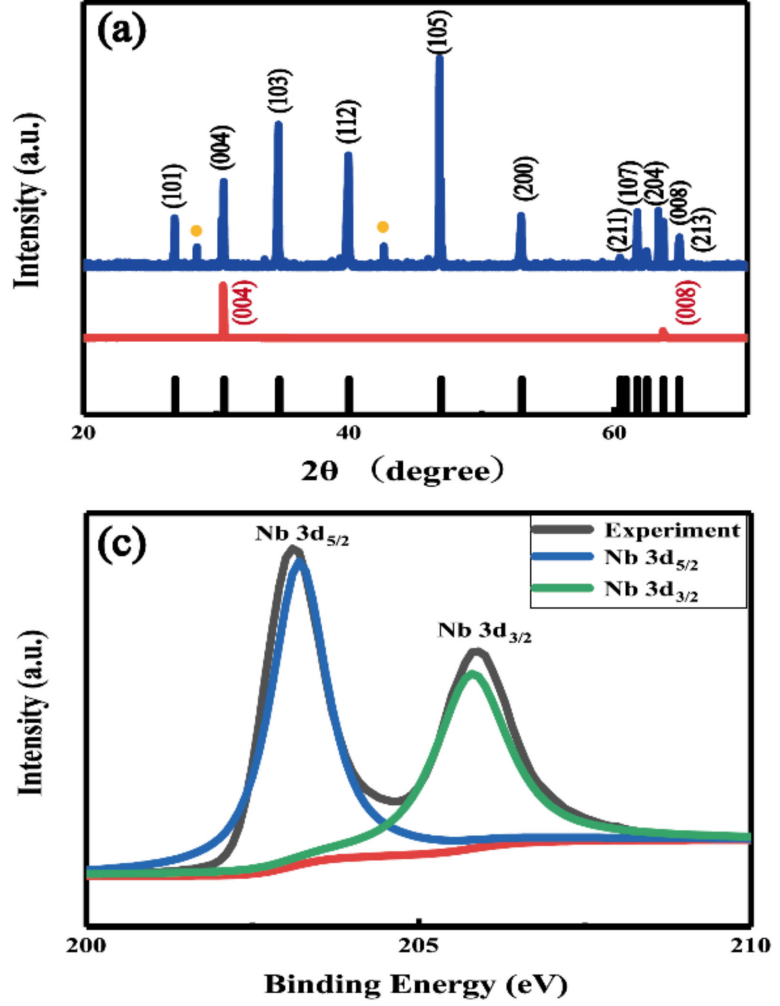
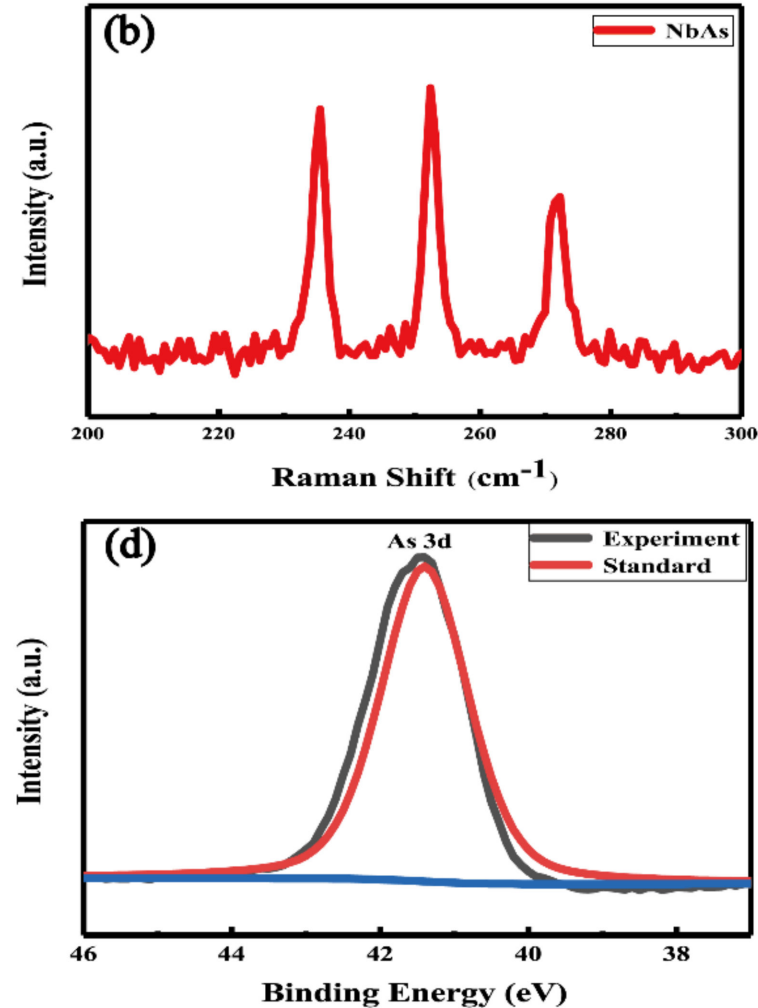

Figure 3. (a) XRD pattern of $\mathrm{NbAs}$ crystal powder. The $\{001\}$ facets of $\mathrm{NbAs}$ single crystal (black line in the figure is PDF card of the $\mathrm{NbAs}$ and diffraction peaks of the polyarsenides marked by yellow dots). (b) Raman spectra of NbAs single crystal. (c,d) Typical XPS spectrum of Nb 3d and As $3 \mathrm{~d}$ core level of $\mathrm{NbAs}$ single crystal.

\subsection{Material Characterization}

As a widely used and powerful characterization method, Raman spectroscopy can preliminarily elucidate the phono information of synthetic crystals [23]. We characterized the $\mathrm{NbAs}$ crystals by Raman spectroscopy, shown in Figure $3 \mathrm{~b}$ for the bulk NbAs crystals, which mainly displayed three prominent Raman peaks in the range of $180-330 \mathrm{~cm}^{-1}$. According to the previous Raman spectra of $\mathrm{NbAs}[24,25]$, we marked the vibration modes corresponding to the three peak positions in Figure $3 \mathrm{~b}$, which are $\mathrm{B}_{1}$ mode at $234 \mathrm{~cm}^{-1}, \mathrm{~B}_{1}$ mode at $252 \mathrm{~cm}^{-1}$, and $A_{1}$ mode at $272 \mathrm{~cm}^{-1}$, respectively. The spectral characterization results are consistent with the theoretical data $[3,26]$, indicating the excellent crystalline quality of the $\mathrm{NbAs}$ crystals.

A typical X-ray photoelectron spectroscopy (XPS) spectrum of NbAs is shown in Figure $3 c, d$. The XRD data show that there may have been a small amount of polyarsenide in the sample. Therefore, we first etched the sample in the XPS test and obtained the XPS of $\mathrm{NbAs}$ after removing the surface layer of $20 \mathrm{~nm}$. Then, the experimental data were labeled by consulting the literature and comparing the database, i.e., $\mathrm{Nb}_{3 / 2}$ at $203.1 \mathrm{eV}$ and $\mathrm{Nb}$ $3 \mathrm{~d}_{3 / 2}$ at $205.8 \mathrm{eV}$. The pair had energy splitting of $\sim 2.7 \mathrm{eV}$ [26], which is consistent with our expectation for the spin-orbit-split $3 \mathrm{~d}_{5 / 2}$ and $3 \mathrm{~d}_{3 / 2}$ levels of $\mathrm{Nb}$ core levels. Similarly, the XPS data of As at the low binding energy end are consistent with the reported data, indicating that $41.5 \mathrm{eV}$ belongs to the $3 \mathrm{~d}$ orbit of As. Again, these are consistent with the XPS results of $\mathrm{NbAs}$ materials reported.

As a means of characterizing crystal quality and structure, atomic morphology characterization can more intuitively and accurately reflect the specific information of $\mathrm{Nb}$ and $\mathrm{As}$ atoms in $\mathrm{NbAs}$ crystals. Figure $4 \mathrm{a}$ is a typical STEM image that shows clear lattice stripes, and Figure $4 \mathrm{~b}$ is a low-power SEM image of bulk NbAs placed on an ultra-thin carbon film. The test results indicate that the spacing between (001) and (010) is approximately $3.4 \AA$, which is consistent with the data in the literature. The diffraction points of (110) and 
(001) are also shown in the Fast Fourier Transform (FFT) diagram in Figure 4a. Similarly, the structure of these facets is shown in Figure $4 \mathrm{~d}$,e. In recent research on $\mathrm{NbAs}$ crystals, the coexistence of hexagonal and tetragonal phases was discussed. The observation or characterization of a $\mathrm{NbAs}$ single crystal on the atomic scale shows that the crystal is a single-phase structure [10].
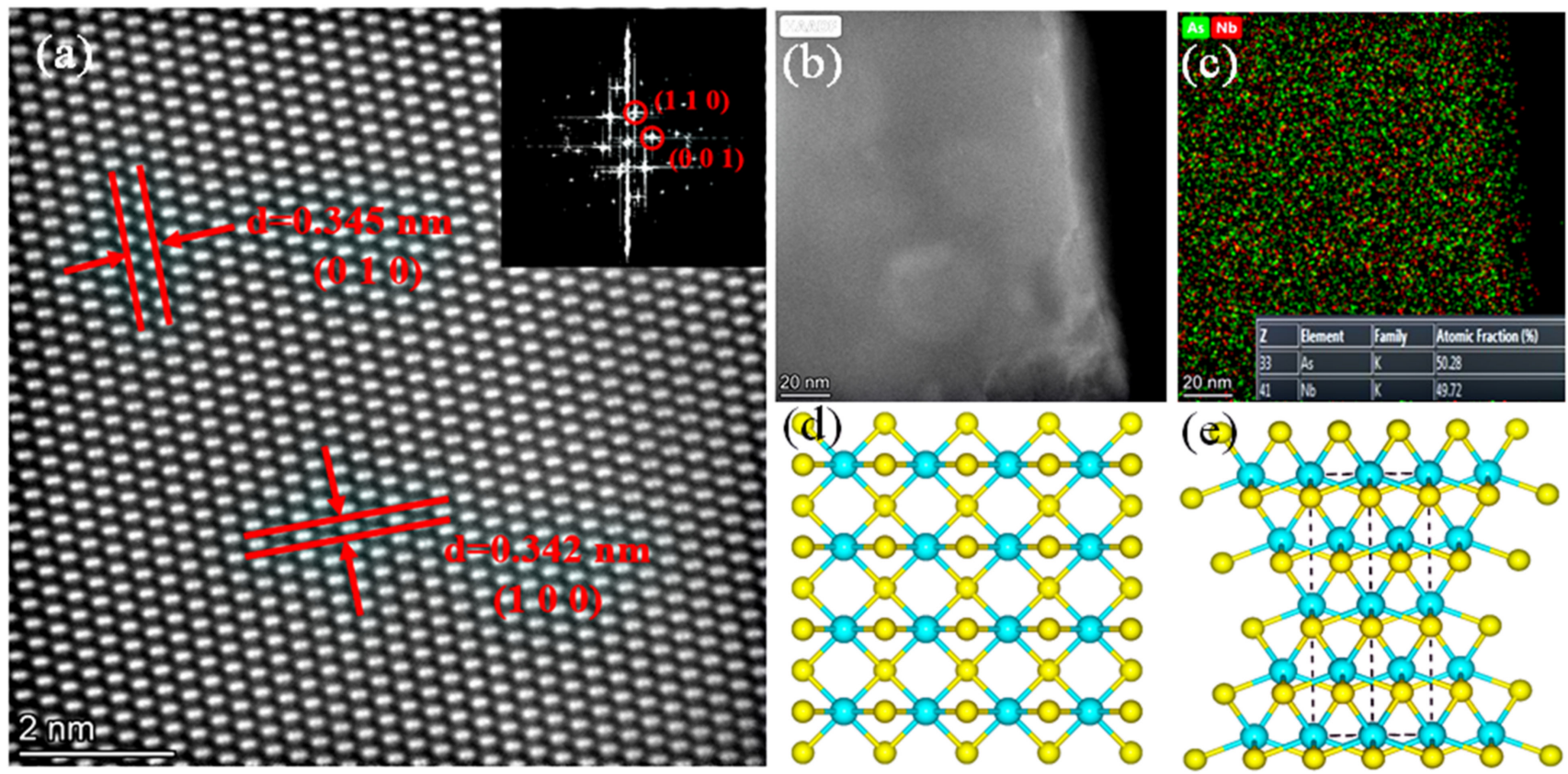

Figure 4. (a) HAASF-STEM images of NbAs single crystal (the illustration is FFT pattern). (b) TEM images of bulk NbAs placed on an ultra-thin carbon film. (c) EDX mapping of bulk NbAs. (d) and (e) show the (001) and (110) facets of $\mathrm{NbAs}$ single crystal.

In our experiments, the coexistence phase problem was overcome by improving the bottom-seed method and synthesizing the high-quality $\mathrm{NbAs}$ crystals with a single-phase structure, highlighting our work's significance. The EDX is shown in Figure 4c, in which the uniform distribution of $\mathrm{Nb}$ and As atoms can be seen. The illustration also indicates that $\mathrm{Nb}$ :As is close to 1:1. This information proves the high quality of our crystals on the micro-scale.

\subsection{Photoelectric Response Detection of $\mathrm{NbAs}$ Crystal}

As for the photoelectric response of $\mathrm{NbAs}$, due to the inherent limitations of the experimental environment, we only investigated the photoelectric detection prototype of Weyl semimetal $\mathrm{NbAs}$ in the visible region (550-800 $\mathrm{nm}$ ) at room temperature.

After evaporation, the tape was carefully torn off, and the area covered by the tape became the device channel of the unevaporated electrode. A schematic diagram of the $\mathrm{Nb}$ As photodetector is shown in Figure 5a. The illustration in Figure 5a shows the schematic diagram of the photoelectron generation principle of $\mathrm{NbAs}$ under light conditions. Under laser irradiation, $\mathrm{NbAs}$ absorbs photons and then causes electron transition to generate carriers, resulting in a photocurrent. With the opening and closing of the incident laser, the current difference between the light condition and the dark condition can be collected. The dark current $\left(\mathrm{I}_{\text {dark }}\right)$ is measured under the conditions of $\mathrm{V}_{\mathrm{DS}}=-0.1$ to $0.1 \mathrm{~V}$ and $\mathrm{V}_{\mathrm{GS}}=0 \mathrm{~V}$. The I-V curve is shown in Figure 5b; the linearity of the curve indicates that the Schottky barrier at the contact interface between the $\mathrm{NbAs}$ sample and the $\mathrm{Cr} / \mathrm{Au}$ electrode is shallow. Figure $5 \mathrm{~b}$ illustrates an $\mathrm{I}-\mathrm{V}$ output curve near $0 \mathrm{~V}$ and $0 \mathrm{~mA}$. As can be seen, when $\mathrm{V}_{\mathrm{DS}}=0 \mathrm{~V}$, the output curve does not entirely intersect with the $\mathrm{X}$-axis $\left(\mathrm{V}_{\mathrm{DS}}=0 \mathrm{~V}\right.$, $\left.\mathrm{I}_{\mathrm{DS}} \approx-0.1 \mathrm{~mA}\right)$. Therefore, we believe that the smoothness of the curve is poor and the actual measured current results at $\mathrm{V}_{\mathrm{DS}}=0 \mathrm{~V}$ are within the reasonable error range, and the 
error is related to the electrode fabrication process, possible surface oxidation, and possible surface thermal effect. The incident light is usually projected on the (001) surface, as shown in the area marked by the red circle in Figure 2.
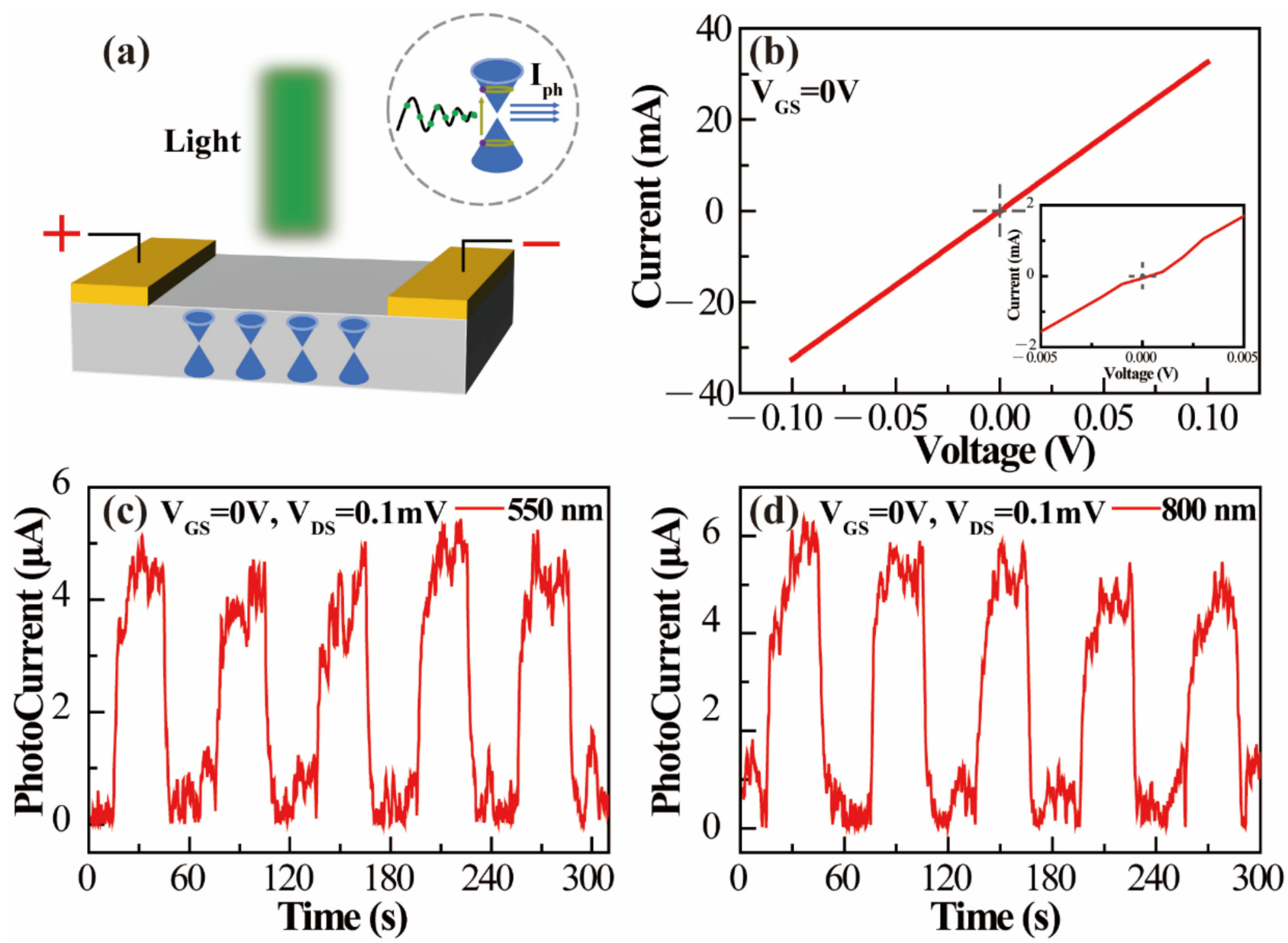

Figure 5. (a) Schematic diagram of $\mathrm{NbAs}$ photoelectric device equipment. Illustration: schematic diagram of photocurrent generation under illumination conditions. (b) The I-V curve of the photodetecting prototype. $(\mathbf{c}, \mathbf{d})$ When the $\mathrm{V}_{\mathrm{DS}}=0.1 \mathrm{mV}, \mathrm{V}_{\mathrm{GS}}=0 \mathrm{~V}$, and the incident light intensity is $10 \mathrm{~mW}$, the I-T curve of the device at $550 \mathrm{~nm}$ and $800 \mathrm{~nm}$ incident light is as shown.

The time-resolved photoelectric response curve of $\mathrm{NbAs}$ was measured the sourcedrain voltage $\mathrm{V}_{\mathrm{DS}}=0.1 \mathrm{mV}$ and gate voltage $\mathrm{V}_{\mathrm{GS}}=0 \mathrm{~V}$. We only list the test results under $550 \mathrm{~nm}$ and $800 \mathrm{~nm}$ lasers, as shown in Figure 5c,d. It can be seen from the time-resolved I-T curve of $\mathrm{NbAs}$ that, under laser conditions, $\mathrm{NbAs}$ exhibits a $\mu \mathrm{A}$-level light response current. Under light and dark conditions, different current outputs show the sensitivity of $\mathrm{NbAs}$ to the laser. In addition, the periodic fast switching of the bright current and dark current at room temperature indicates that $\mathrm{NbAs}$ has a strong absorption capacity for photons under laser irradiation. This process highlights the strong photon absorption of $\mathrm{NbAs}$ crystals in the visible region and at room temperature, indicating the advantages of $\mathrm{NbAs}$ in the field of photoelectric detection. By comparison, the light-dark current switching ratio is close to 5, but the light-dark current is relatively poor under stable conditions. Therefore, we believe that the electrode fabrication method may affect the test results. In addition, the laser has poor stability at $10 \mathrm{~mW}$ (if the laser intensity is too low, the photoresponse current of $\mu \mathrm{A}$ magnitude will be more difficult to distinguish) and cannot achieve a stable, high-intensity laser output, and this may cause the instability of the photoresponse current. 
Although stable test conditions cannot be achieved, the photoelectric response current of more than $\mu \mathrm{A}$ magnitude only at $0.1 \mathrm{mV}$ indicates the research potential of $\mathrm{NbAs}$ in photoelectric response detection $[6,27,28]$.

The photoelectric responsivity $(R)$ and detection rate $\left(D^{*}\right)$ can be defined as follows [28-32]:

$$
R=\frac{I_{p h}}{P A}
$$

Among them, $I_{p h}, P$, and $A$ represent the photocurrent, incident optical power density, and the effective irradiation area of the detector.

$$
D^{*}=R \sqrt{\frac{S}{2 q I_{d a r k}}}
$$

where, $R, S, q$, and $I_{\text {dark }}$ represent the responsivity, the effective irradiation area of the heterojunction, the primary charge, and the dark current, respectively. Figure $6 \mathrm{a}, \mathrm{b}$ list the device's $R$ and $D^{*}$ related information in the range of 550-800 $\mathrm{nm}$ (corresponding to the incident photon energy of 1.5 to $2.25 \mathrm{eV}$ ) when the incident power is fixed at $10 \mathrm{~mW}$. It can be seen that $R$ and $D^{*}$ do not change significantly with the incident photon energy. Except for the high responsivity at $550 \mathrm{~nm}$, the responsivity is stable at $2 \mathrm{~mA} / \mathrm{W}\left(D^{*}\right.$ close to $10^{7}$ Jones). When the incident laser wavelength is $550 \mathrm{~nm}, R$ and $D^{*}$ reach $16 \mathrm{~mA} / \mathrm{W}$ and $1.1 \times 10^{8}$ Jones, respectively. This result provides clues as to the light response mechanism. It shows that $\mathrm{NbAs}$ has stronger absorption and more energy scattering events at short wavelengths, which helps to achieve a more significant light response. In addition, significantly more energy is transferred from the absorbed photons to the electrons excited on the short-wavelength side.
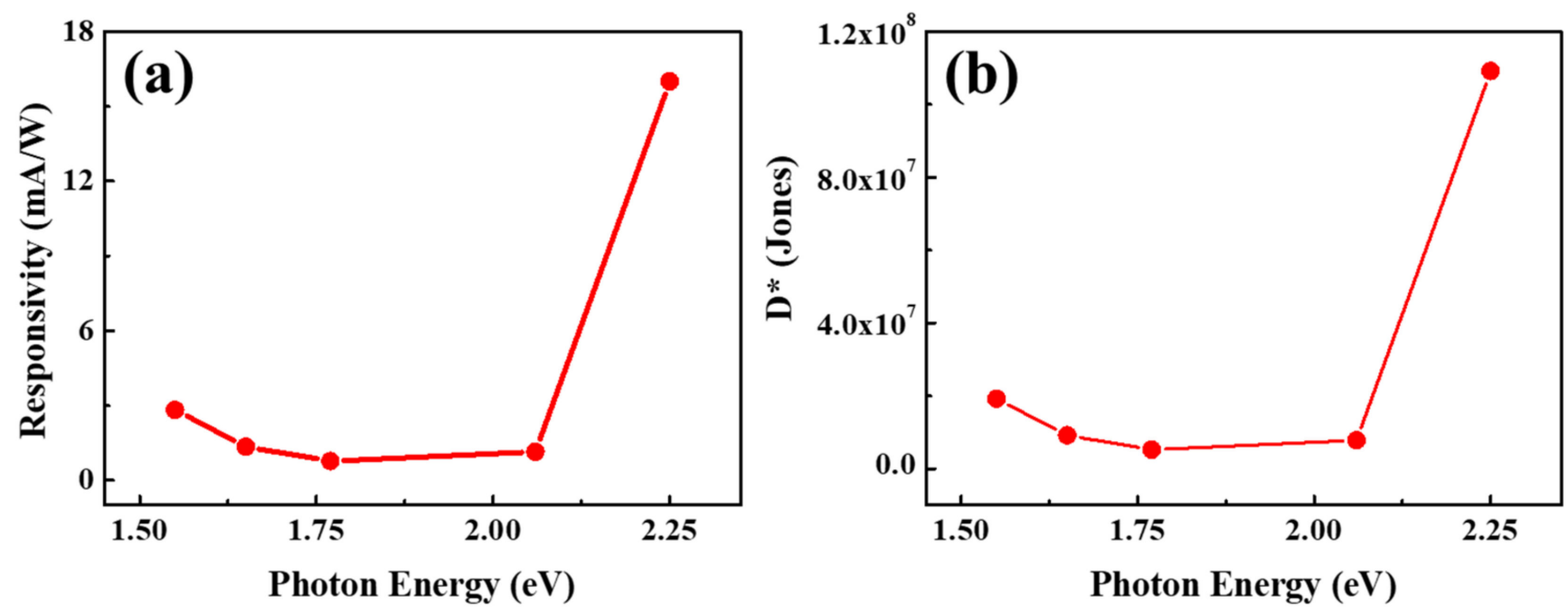

Figure 6. (a) Responsivity variation characteristics of $\mathrm{NbAs}$ photoelectric device prototype within 1.5 to $2.25 \mathrm{eV}$. (b) The relationship between the detection rate $D^{*}$ of $\mathrm{NbAs}$ devices and the energy of incident photons.

It is worth noting that, according to reports [6], TaAs has a broad-spectrum light response from the visible range to the long-infrared range at room temperature. As for $\mathrm{NbAs}$, we only conducted preliminary tests on its light response in the visible light range, and its broadband light response at room temperature still needs more in-depth theoretical and experimental research.

\section{Conclusions}

In summary, the one-dimensional size of the $\mathrm{NbAs}$ single crystal grown by the CVT method using the bottom-seed method can reach $4 \mathrm{~mm}$, and the three-dimensional size can 
reach $4 \times 3 \times 3 \mathrm{~mm}^{3}$. The quality of the product has been confirmed by powder $\mathrm{XRD}$ and Raman methods. More importantly, the atomic-level morphology characterization results show that we have overcome the multi-phase coexistence in the past growth process. XPS data explain that the surface state of $\mathrm{NbAs}$ crystals will be affected by oxygen and lead to some changes, as specific results need to be further studied and discussed. In addition, we prepared a prototype of the $\mathrm{NbAs}$ photodetector in the visible light range and discussed the relationship between $R$ and $D^{*}$ with the energy of incident photons. Our improved growth method provides a reference and helps in the growth of difficult-to-synthesize materials such as Weyl semimetal. At the same time, the synthesis of high-quality crystals guarantees the subsequent electrical properties research and photoelectric performance detection of this type of Weyl semimetal. Due to the inherent limitations of the experimental conditions, we only obtained the relevant information of the time-resolved response of the $\mathrm{NbAs}$ photodetector in the visible range and discussed the relationship between $R$, $D^{*}$, and the incident photon energy. By comparison, it was found that the test results of $\mathrm{NbAs}$ were similar to those of TaAs photodetectors in the visible range. In addition, the photoresponse current at $\mu \mathrm{A}$ level at room temperature indicated that $\mathrm{NbAs}$ has a reasonable photon absorption rate. In this experiment, we only fabricated a relatively primary $\mathrm{NbAs}$ optoelectronic device. With the help of the strong interaction between $\mathrm{NbAs}$ and light, the optimization design of the device will further improve the light response and then highlight the light response advantages of the Weyl semimetal in a wide range at room temperature. Excellent photon absorption and ambient temperature at room temperature indicate the potential of the Weyl semimetal in photoelectric detection.

Author Contributions: Methodology, Y.S.; validation, B.Z., Y.S., Y.X. and Z.H. (Zongju Huo); investigation, B.Z.; data curation, Y.S.; writing-original draft preparation, H.Q.; writing-review and editing, H.L.; project administration, Z.H. (Zhanggui Hu). All authors have read and agreed to the published version of the manuscript.

Funding: This research was funded by the NSFC $(51972229,51802218,51872198,52172151)$, the Natural Science Foundation of Tianjin (19JCYBJC17800), and the National Defense Science and Technology 173 Program (2021-JCJQ-JJ-0639).

Institutional Review Board Statement: Not applicable.

Informed Consent Statement: Not applicable.

Data Availability Statement: Not applicable.

Acknowledgments: The authors thank Wen Cheng Ma and Lu Jie Li for the technical support.

Conflicts of Interest: The authors declare that they have no conflicts of interest.

\section{References}

1. Xu, S.-Y.; Alidoust, N.; Belopolski, I.; Yuan, Z.; Bian, G.; Chang, T.-R.; Zheng, H.; Strocov, V.N.; Sanchez, D.S.; Chang, G.; et al. Discovery of a Weyl fermion state with Fermi arcs in niobium arsenide. Nat. Phys. 2015, 11, 748-754. [CrossRef]

2. Huang, S.M.; Xu, S.Y.; Belopolski, I.; Lee, C.C.; Chang, G.; Chang, T.R.; Wang, B.; Alidoust, N.; Bian, G.; Neupane, M.; et al. New type of Weyl semimetal with quadratic double Weyl fermions. Proc. Natl. Acad. Sci. USA 2016, 113, 1180-1185. [CrossRef] [PubMed]

3. Yuan, X.; Zhang, C.; Zhang, Y.; Yan, Z.; Lyu, T.; Zhang, M.; Li, Z.; Song, C.; Zhao, M.; Leng, P.; et al. The discovery of dynamic chiral anomaly in a Weyl semimetal NbAs. Nat. Commun. 2020, 11, 1259. [CrossRef] [PubMed]

4. Xu, Q.; Zhang, Y.; Koepernik, K.; Shi, W.; Van den Brink, J.; Felser, C.; Sun, Y. Comprehensive scan for nonmagnetic Weyl semimetals with nonlinear optical response. Npj Comput. Mater. 2020, 6, 1-7. [CrossRef]

5. Ji, Z.; Liu, G.; Addison, Z.; Liu, W.; Yu, P.; Gao, H.; Liu, Z.; Rappe, A.M.; Kane, C.L.; Mele, E.J.; et al. Spatially dispersive circular photogalvanic effect in a Weyl semimetal. Nat. Mater. 2019, 18, 955-962. [CrossRef]

6. Chi, S.; Li, Z.; Xie, Y.; Zhao, Y.; Wang, Z.; Li, L.; Yu, H.; Wang, G.; Weng, H.; Zhang, H.; et al. A Wide-Range Photosensitive Weyl Semimetal Single Crystal-TaAs. Adv. Mater. 2018, 30, 1801372. [CrossRef]

7. Shekhar, C.; Nayak, A.K.; Sun, Y.; Schmidt, M.; Nicklas, M.; Leermakers, I.; Zeitler, U.; Skourski, Y.; Wosnitza, J.; Liu, Z.; et al. Extremely large magnetoresistance and ultrahigh mobility in the topological Weyl semimetal candidate NbP. Nat. Phys. 2015, 11, 645-649. [CrossRef] 
8. Yuan, X.; Yan, Z.; Song, C.; Zhang, M.; Li, Z.; Zhang, C.; Liu, Y.; Wang, W.; Zhao, M.; Lin, Z.; et al. Chiral Landau levels in Weyl semimetal $\mathrm{NbAs}$ with multiple topological carriers. Nat. Commun. 2018, 9, 1854. [CrossRef]

9. Osterhoudt, G.B.; Diebel, L.K.; Gray, M.J.; Yang, X.; Stanco, J.; Huang, X.; Shen, B.; Ni, N.; Moll, P.J.W.; Ran, Y.; et al. Colossal mid-infrared bulk photovoltaic effect in a type-I Weyl semimetal. Nat. Mater. 2019, 18, 471-475. [CrossRef]

10. Guo, C.; Tian, H.F.; Yang, H.X.; Sun, K.; Wei, L.L.; Chen, G.F.; Li, J.Q. Hexagonal Phase Intergrown with the Tetragonal Weyl Semimetal TaAs. Cryst. Growth Des. 2017, 17, 1747-1751. [CrossRef]

11. Boller, H.; Parthe, E. The Transposition Structure of NbAs and of Similar Monophosphides. Acta Cryst. 1963, 1963. 16, 1095. [CrossRef]

12. Li, Z.; Chen, H.; Jin, S.; Gan, D.; Wang, W.; Guo, L. Chen, X. Weyl Semimetal TaAs: Crystal Growth, Morphology, and Thermodynamics. Cryst. Growth Des. 2016, 16, 1172-1175. [CrossRef]

13. Sapkota, D.; Mukherjee, R.; Mandrus, D. Single Crystal Growth, Resistivity, and Electronic Structure of the Weyl Semimetals NbP and TaP. Crystals 2016, 6, 160. [CrossRef]

14. Lin, D.Y.; Guo, B.C.; Dai, Z.Y.; Lin, C.F.; Hsu, H.P. PbI 2 Single Crystal Growth and Its Optical Property Study. Crystals 2019,9 , 589. [CrossRef]

15. Zhu, P.; Li, Y.; Yang, X.; Yang, Y.; Zhang, X.; Lin, X.; Yang, F.; Li, X.; Wang, Z. Synthesis of Superconducting InxSn1-xTe $(0.04<\mathrm{x}<0.1)$ Large Single Crystal by Liquid Transport Method. Crystals 2021, 11, 474.

16. Panella, J.R.; Trump, B.A.; Marcus, G.G.; McQueen, T.M. Seeded Chemical Vapor Transport Growth of Cu2OSeO3. Cryst. Growth Des. 2017, 17, 4944-4948. [CrossRef]

17. Selter, S.; Shemerliuk, Y.; Büchner, B.; Aswartham, S. Crystal Growth of the Quasi-2D Quarternary Compound AgCrP2S6 by Chemical Vapor Transport. Crystals 2021, 11, 500. [CrossRef]

18. Sun, Z.; Liufu, S.; Chen, X.; Chen, L. Enhanced thermoelectric properties of $\mathrm{Bi}_{0.5} \mathrm{Sb}_{1.5} \mathrm{Te}_{3}$ films by chemical vapor transport process. ACS Appl Mater Interfaces 2011, 3, 1390-1393. [CrossRef]

19. Saini, G.S.; Calvert, L.D.; Taylor, J.B. Preparation and Characterization of Crystals of MX- and MX 2 -Type of Niobium and Tantalum. Can. J. Chem. 1964, 42, 630-634. [CrossRef]

20. Zhang, M.D.; Hou, X.Y.; Wang, Q.; Wang, Y.Y.; Zhao, L.X.; Wang, Z.; Gu, Y.D.; Zhang, F.; Xia, T.L.; Ren, Z.A.; et al. Tip-induced superconductivity on the topological semimetals $\mathrm{TaAs}_{2}$ and NbAs 2 . Phys. Rev. B 2020, 102, 085139. [CrossRef]

21. Wang, Y.Y.; Yu, Q.H.; Guo, P.J.; Liu, K.; Xia, T.L. Resistivity plateau and extremely large magnetoresistance in $\mathrm{NbAs}_{2}$ and TaAs 2 Phys. Rev. B 2016, 94, 041103(R). [CrossRef]

22. Peramaiyan, G.; Sankar, R.; Muthuselvam, I.P.; Lee, W.L. Anisotropic magnetotransport and extremely large magnetoresistance in $\mathrm{NbAs}_{2}$ single crystals. Sci. Rep. 2018, 8, 6414. [CrossRef] [PubMed]

23. Greco, S.; Dal Zilio, S.; Bek, A.; Lazzarino, M.; Naumenko, D. Frequency Modulated Raman Spectroscopy. ACS Photonics 2017, 5 , 312-317. [CrossRef]

24. Liu, H.W.; Richard, P.; Song, Z.D.; Zhao, L.X.; Chen, G.F.; Ding, H. Raman study of lattice dynamics in the Weyl semimetal TaAs. Phys. Rev. B 2015, 92, 064302. [CrossRef]

25. Chiarello, G.; Hofmann, J.; Li, Z.; Fabio, V.; Guo, L.; Chen, X.; Das Sarma, S.; Politano, A. Tunable surface plasmons in Weyl semimetals TaAs and NbAs. Phys. Rev. B 2019, 99, 121401. [CrossRef]

26. Liu, H.W.; Zhang, G.H.; Richard, P.; Zhao, L.X.; Chen, G.G.; Ding, H. Spatially Resolved X-ray Photoemission Electron Microscopy of Weyl Semimetal NbAs. Cryst. Growth Des. 2018, 18, 5210-5213. [CrossRef]

27. Bedoya-Pinto, A.; Pandeya, A.K.; Liu, D.; Deniz, H.; Chang, K.; Tan, H.; Han, H.; Jena, J.; Kostanovskiy, I.; Parkin, S.S.P. Realization of Epitaxial NbP and TaP Weyl Semimetal Thin Films. ACS Nano 2020, 14, 4405-4413. [CrossRef]

28. Fedotov, A.; Shendyukov, V.; Tsybulskaya, L.; Perevoznikov, S.; Dong, M.; Xue, X.; Feng, X.; Sayyed, M.I.; Zubar, T.; Trukhanov, A.; et al. Electrodeposition conditions-dependent crystal structure, morphology and electronic properties of Bi films. J. Alloy. Compd. 2021, 887, 161451. [CrossRef]

29. Ross, J.S.; Klement, P.; Jones, A.M.; Ghimire, N.J.; Yan, J.; Mandrus, D.G.; Taniguchi, T.; Watanabe, K.; Kitamura, K.; Yao, W.; et al Electrically tunable excitonic light-emitting diodes based on monolayer WSe 2 p-n junctions. Nat. Nanotechnol. 2014, 9, 268-272. [CrossRef]

30. Pezeshki, A.; Shokouh, S.H.; Nazari, T.; Oh, K.; Im, S. Electric and Photovoltaic Behavior of a Few-Layer $\alpha-\mathrm{MoTe}_{2} / \mathrm{MoS}_{2}$ Dichalcogenide Heterojunction. Adv. Mater. 2016, 28, 3216-3222. [CrossRef]

31. Zhang, S.; Guo, S.; Huang, Y.; Zhu, Z.; Cai, B.; Xie, M.; Zhou, W.; Zeng, H. Two-dimensional SiP: An unexplored direct bandgap semiconductor. 2D Mater. 2016, 4, 015030. [CrossRef]

32. Jing, Y.; Ma, Y.; Li, Y.; Heine, T. GeP 3 : A Small Indirect Band Gap 2D Crystal with High Carrier Mobility and Strong Interlayer Quantum Confinement. Nano Lett. 2017, 17, 1833-1838. [CrossRef] 